\title{
Secondary structures of hyaluronate and chondroitin sulphates
}

\author{
$A^{1}$ H n.m.r. study of $\mathrm{NH}$ signals in dimethyl sulphoxide solution
}

\begin{abstract}
John E. SCOTT, ${ }^{*}$ Frank HEATLEY, $\dagger$ David MOORCROFT $\dagger$ and Anthony H. OLAVESEN $\ddagger$ *Biochemical Morphology, Department of Medical Biochemistry, Medical School, University of Manchester, Manchester M13 9PT, U.K., †Department of Chemistry, University of Manchester, Manchester M13 9PT, U.K., and $\ddagger$ Department of Biochemistry, University College, Cardiff CF1 1XL, Wales, U.K.
\end{abstract}

\section{(Received 11 August 1981/Accepted 18 September 1981)}

\begin{abstract}
${ }^{1} \mathrm{H}$ n.m.r. spectra in $\left[{ }^{2} \mathrm{H}_{6}\right]$ dimethyl sulphoxide of dodecyltrimethylammonium salts of chondroitin sulphates and hyaluronate, or sodium salts of oligomers from hyaluronate, showed unambiguous $\mathrm{NH}$ signals. The acetamido $\mathrm{NH}$ occurs in two different environments: environment I ('normal') in simple sugars, and environment II (hydrogen-bonded $\mathrm{NH}$ ) appearing in tri- or tetrasaccharides, indicating a secondary structure in hyaluronate (and some chondroitin sulphates) involving a hydrogen-bonded acetamido $\mathrm{NH}$.
\end{abstract}

The observation that certain glycosaminoglycuronans were oxidized by periodate at very much lower rates than those expected from model glucuronides led to the proposal that they contained intramolecular secondary structures involving hydrogen-bonded acetamido groups (Scott \& Tigwell, 1975, 1978). The acetamido group is bifunctional, acting as donor and acceptor to groups on neighbouring sugars, so that three sugar units are involved in the proposed structures. Model-building supported the conclusions, which were further extended by computer calculations (Atkins et al., 1980). Only hyaluronate and the chondroitin sulphates possess the necessary steric arrangements of donor and acceptor groups that could lead to these hydrogen-bonded arrays.

Possible biological implications of the secondary structures have been discussed (Scott, 1980; Scott et al., 1981).

An n.m.r. study in ${ }^{2} \mathrm{H}_{2} \mathrm{O}$ showed that the behaviour of the methyl signal of the acetamido group in a series of polymers and oligomers was consistent with the proposed structures (Scott \& Heatley, 1979). Groups directly involved ( $\mathrm{OH}$ and $\mathrm{NH}$ ) could not be observed because of rapid exchange of protons with the solvent. It is desirable that studies should be conducted in a solvent in which exchange cannot occur, e.g. dimethyl sulphoxide. Acetamido sugars, sodium salts of uronic acids and the component disaccharides of the polymers are sufficiently soluble in dimethyl sulphoxide to enable n.m.r. studies to be performed easily (Heatley et al., 1979). All the relevant ${ }^{1} \mathrm{H}$ signals were identified (Heatley et al., 1981). Unfortu- nately, the sodium salts of the polymers themselves are much less soluble in dimethyl sulphoxide. Quaternary ammonium salts of polyanions are soluble in some organic solvents (Scott, 1960). We found that dodecyltrimethylammonium salts of hyaluronate, chondrotin sulphates etc. are soluble at quite high $(5 \%, w / v)$ concentrations in dimethyl sulphoxide, thus permitting a comparison of the $\mathrm{NH}$ frequencies in several polymers with those from monomeric sugars, disaccharides and higher oligomers.

Some of the present results have been communicated preliminarily (Scott et al., 1977).

\section{Experimental}

Materials

The polymers used were those described previously (Scott \& Heatley, 1979). The disaccharides and monomeric sugars were those described by Heatley et al. $(1979,1981)$.

\section{Quaternary ammonium salts of polyanions}

Aqueous solutions of polyanions $(0.5 \mathrm{ml}$ portions) were titrated with aqueous dodecyltrimethylammonium bromide $(1.0 \mathrm{mg} / \mathrm{ml})$ to a flocculent end point, corresponding to the stoicheiometric formation of the insoluble quaternary ammonium salt (Scott, 1960). More-concentrated solutions of polyanions (approx. $10 \mathrm{mg} / \mathrm{ml}$ ) were then mixed with the appropriate amount of quaternary ammonium bromide, based on titration. The flocculent precipitates were centrifuged, washed several times with 
distilled water, and dried for several days in vacuo over $\mathrm{P}_{2} \mathrm{O}_{5}$.

\section{Oligosaccharides from hyaluronate}

Hyaluronate and crude bovine hyaluronidase (350 W.H.O. turbidity units/mg; Miles Laboratories; $1 \mathrm{mg} / 10 \mathrm{mg}$ of hyaluronate) were incubated together in $0.1 \mathrm{M}$-sodium acetate buffer, $\mathrm{pH} 5.2$, containing $0.15 \mathrm{M}-\mathrm{NaCl}$ at $37^{\circ} \mathrm{C}$ for $24 \mathrm{~h}$ under toluene. A further amount of hyaluronidase $(1 \mathrm{mg}$ of enzyme $/ 10 \mathrm{mg}$ of substrate) was added, and $24 \mathrm{~h}$ later the reaction was stopped by heating at $100^{\circ} \mathrm{C}$ for $15 \mathrm{~min}$. The solution was clarified by centrifugation, and concentrated in vacuo at $37^{\circ} \mathrm{C}$.

The oligosaccharides were applied to a column of Sephadex G-25 (fine grade) equilibrated in $0.1 \mathrm{M}$ $\mathrm{NaCl}$. Fractions were analysed for uronic acid by the carbazole method (Bitter \& Muir, 1962). Peaks containing uronate were desalted on Sephadex G-25 (medium grade), and freeze-dried. Oligosaccharides analysed for reducing $\mathrm{N}$-acetylglucosamine by the method of Reissig et al. (1955) showed ratios to uronate of $3.1: 1$ (hexasaccharide) and 4.02:1 (octasaccharide).

N.m.r. experiments

${ }^{1} \mathrm{H}$ n.m.r. spectra were run on a Varian SC 300 spectrometer operating at $300 \mathrm{MHz}$. Solutions were prepared in $\left[{ }^{2} \mathrm{H}_{6}\right]$ dimethyl sulphoxide.

\section{Results}

Dodecyltrimethylammonium salts of chondroitin sulphates and hyaluronate were soluble at concentrations of $50 \mathrm{~g} / 1$ in $\left[{ }^{2} \mathrm{H}_{6}\right]$ dimethyl sulphoxide at room temperature. Slight warming speeded dissolution.

Spectra in $\left[{ }^{2} \mathrm{H}_{6}\right]$ dimethyl sulphoxide of acetamido sugars, disaccharides (e.g. $N$-acetylchondrosinate, hyalobiuronate and derivatives) showed clearly resolved and sharp signals. Those from tetrahexa- and octa-saccharides from hyaluronate were less sharp, but still very clear. Spectra from polymer solutions showed much broader signals. Contributions from dodecyltrimethylammonium and ${ }^{1} \mathrm{H}^{2} \mathrm{HO}$ obscured parts of the polysaccharide spectrum, but peaks to low field $(\delta>6$ p.p.m.) were not subject to interference.

Table 1 shows chemical shifts of NH signals. The $\mathrm{NH}$ resonance at about $\delta=7.8$ p.p.m. in monosaccharides and synthetic disaccharides has been attributed unambiguously (Heatley et al., 1981). The synthetic disaccharides consist solely of $\alpha$-anomer, and only one NH peak was present. In the spectra of

Table 1. Chemical shifts $(\delta)$ in $\left[{ }^{2} H\right]$ dimethyl sulphoxide of $N H$ in 2-deoxy-2-acetamidohexose-containing sugars, disaccharides, oligomers and polymers. of the hyaluronate, chondroitin sulphate and dermatan sulphate series

$N$-Acetylglucosamine

$N$-Acetylgalactosamine*

$N$-Acetylchondrosinate $\ddagger$

Allyl 2-acetamido-2-deoxy-3- $O$-( $\beta$-D-glucopyranosyluronate)a-D-glucopyranoside $† \ddagger$

Methyl 2-acetamido-2-deoxy-3- $O$-( $\beta$-D-glucopyranosyluronate)$\alpha$-D-glucopyranoside $\ddagger$

Methyl 2-acetamido-2-deoxy-3-O-(2,3-di- $O$-methyl- $\beta$-Dglucopyranosyluronate)-4,6-di- $O$-methyl- $\alpha$-D-glucopyranoside $† \ddagger$

Hyaluronate tetrasaccharide $\ddagger$

Hyaluronate hexasaccharide $\ddagger$

Hyaluronate octasaccharide $\ddagger$

Hyaluronate $\S$

Chondroitin 4-sulphate $\S$

Chondroitin 6-sulphate $\S$

Chondroitin sulphate D§॥

Dermatan sulphate§

\begin{tabular}{|c|c|c|}
\hline \multicolumn{3}{|c|}{ Chemical shift (p.p.m.) } \\
\hline \multicolumn{2}{|c|}{ Resonance I } & Resonance II \\
\hline$\alpha$-Anomer & $\beta$-Anomer & \\
\hline $\begin{array}{l}7.67 \\
7.58 \\
7.69 \\
7.82\end{array}$ & 7.79 & \\
\hline
\end{tabular}

7.82

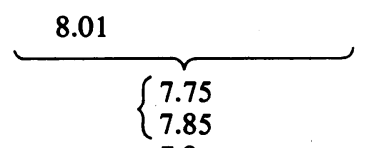

7.8

9.3

9.3

9.3

9.3

8.25

8.65

8.75

8.0

* Data from Schamper (1974).

† Data from Heatley et al. (1981).

$\ddagger$ Sodium salts.

$\S$ Dodecyltrimethylammonium salts.

II Chondroitin D is oversulphated chondroitin sulphate from shark cartilage. 
oligomers prepared by enzymic hydrolysis all $\mathrm{NH}$ peaks were double, one associated with the $\alpha$ anomer and the other with the $\beta$-anomer. Their separation was small, about $\delta=0.1$ p.p.m.

In contrast with the compact group of $\mathrm{NH}$ signals in simple sugars and disaccharides, two groups of $\mathrm{NH}$ resonances were observed in tetra-, hexa- and octa-saccharides from hyaluronate. The high-field peak was at $\delta=7.8$ p.p.m., i.e. the same shift as the NH resonance in simple sugars, whereas the low-field peak was at $\delta=9.3$ p.p.m. The latter peak intensity increased stepwise in ratio to that of the former, from approximately $1: 1$ in the tetrasaccharide to $2: 1$ in the hexasaccharide and $3: 1$ in the octasaccharide.

The polymer NH resonances were to lower field than those of the simple sugars. The shift varied from $\delta=8.0-8.1$ p.p.m. (dermatan sulphate) to $\delta=9.3$ p.p.m. (hyaluronate) (see Table 1).

\section{Discussion}

Resonances in the spectra of oligomers and polymers were assigned to $\mathrm{NH}$ on the basis of: $(a)$ their disappearance on deuteration; $(b)$ their characteristic shifts to low field; $(c)$ their analogy to those of simple acetamido sugars; $(d)$ decoupling experiments, which showed that the NH signals were all coupled to resonances at the shift expected for $\mathrm{H}-2$ in the amino sugar ring.

Our results demonstrate that the acetamido $\mathrm{NH}$ group in the glycosaminoglycuronans can be found in the following two different environments. Environment (I) is characterized by Resonance I at, or very close to $\delta=7.8$ p.p.m., found in monosaccharides, synthetic disaccharides and simple amides. It seems that is a 'normal', non-interacting, amide NH. Environment (II) is characterized by Resonance II up to $\delta=1.5$ p.p.m. to low field of Resonance I. This type of $\mathrm{NH}$ is the only one present in hyaluronate and the chondroitin sulphates. Since both environments (I) and (II) are present in hyaluronate tetrasaccharide and higher oligomers, but environment (II) is absent from the simple sugars and disaccharides, it follows that environment (II) is critically dependent on molecular size, appearing in the series either at the tri- or tetra-saccharide level.

A structure that accounts for these results is that proposed by Scott \& Tigwell $(1975,1978)$, in which the acetamido residue is hydrogen-bonded via $\mathrm{C}=\mathrm{O}$ and $-\mathrm{NH}$ groups to the sugars on each side (Fig. 1). A trisaccharide unit, consisting of two uronate residues $(G)$ and a central hexosamine $\left(N^{\prime}\right)$, is the new 'repeating unit' of the polymer. Thus the disaccharide GN would not contain a hydrogenbonded NH group, whereas the tetrasaccharide GN'GN would (i.e. as part of $N^{\prime}$ ) along with a 'normal' $\mathrm{NH}$, as part of $\mathrm{N}$. The integrated intensities of the two NH signals should be $1: 1$, as observed (see the Results section). Similarly, the ratio of the intensities of the NH signals of the hexa- and octa-saccharides. GN'GN'GN and $\mathrm{GN}^{\prime} \mathrm{GN}^{\prime} \mathrm{GN}^{\prime} \mathrm{GN}$ are $2: 1$ and $3: 1$, as required by the structure (Fig. 1). In the polymers, the proportion of the 'end group' GN would be too small (if it were present) to be detected, and only the signal from $\mathbf{N}^{\prime}$ is observed, in hyaluronate and the chondroitin sulphates.

The attribution of Resonance II to $\mathrm{N}^{\prime}$ is supported $(a)$ by the fact that hydrogen-bonding usually results in movement of the resonance to low field, as compared with similar non-hydrogen-bonded groups (i.e. in $\mathrm{N}$ ), and (b) by the lower rate of disappearance of Resonance II on deuteration (see the Results section), characteristic of more-strongly hydrogen-bonded groups.

Our evidence that a structure involving hydrogen-bonded $\mathrm{NH}$ appears either at the tri- or

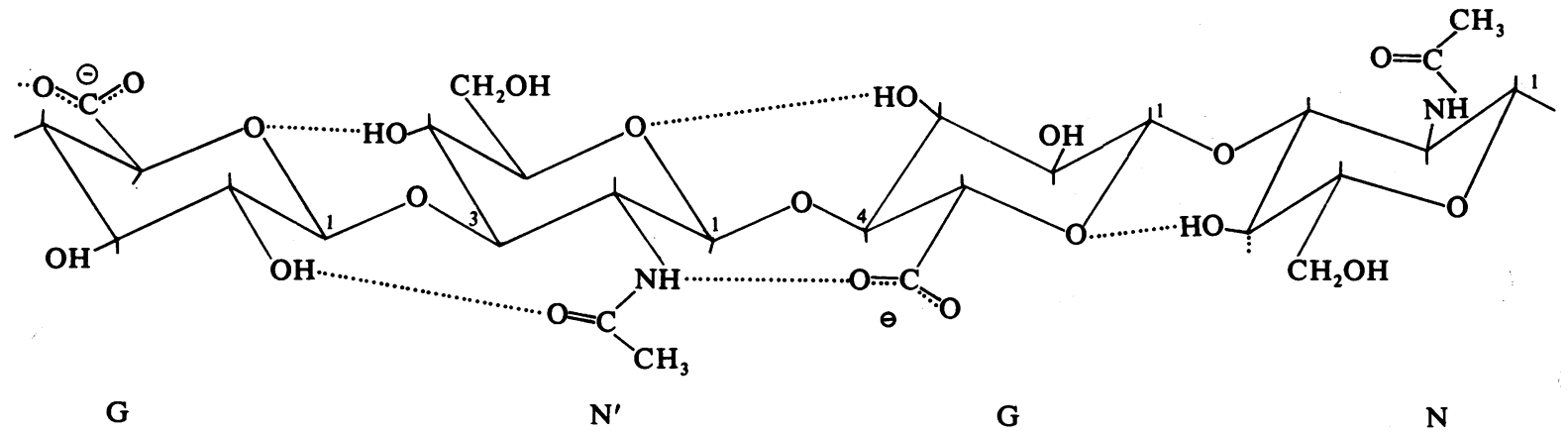

Fig. 1. Structure of a tetrasaccharide segment of hyaluronate, showing four different hydrogen bonds (dotted lines) (based on Atkins et al. (1980]]

Key: G, glucuronate; $\mathrm{N}^{\prime}, N$-acetylglucosamine with a doubly-hydrogen-bonded acetamido group; $\mathrm{N}, N$-acetylglucosamine (in a terminal position) with no hydrogen bond at the acetamido NH. 
tetra-saccharide level should be viewed with the findings made by Cowman et al. (1981), that the c.d. of hyaluronate tetrasaccharide and higher oligomers was equivalent to that of the high polymer, but different from that of the component monosaccharides. On this and similar evidence Cowman et al. (1981) suggested a close approach of $-\mathrm{CO}_{2}^{-}$to the acetamido groups in the polymer. Their results, which were obtained on aqueous solutions, can be interpreted in favour of the structure shown in Fig. 1.

The downfield position of Resonance II in the spectra of chondroitin sulphates and (particularly) hyaluronate suggests that their $\mathrm{NH}$ groups are hydrogen-bonded. The $\delta$ value for NH of dermatan sulphate is only slightly higher than that of the simple sugars, suggesting only weak hydrogenbonding, if any. These findings are also compatible with the structures proposed previously (Scott \& Tigwell, 1975, 1978), similar to Fig. 1. The hydrogen-bonded array requires that the participating ${ }_{-} \mathrm{CO}_{2}^{-}$, acetamido and $\mathrm{C}_{(2)} \mathrm{OH}$ groups must all be equatorial, as must the glycosidic bonds between sugars. Hyaluronate and the chondroitin sulphates fulfil these conditions, whereas dermatan sulphate does not. Depending on whether iduronate in dermatan sulphate is in the ${ }^{4} \mathrm{C}_{1}$ or the ${ }^{1} \mathrm{C}_{4}$ chain conformation, either the $-\mathrm{CO}_{2}^{-}$or iduronidic bond is axial, and the hydrogen-bonded array cannot form.

The considerable downfield shift of Resonance II of hyaluronate, compared with that of the chondroitin sulphates, suggests a correspondingly more stable hydrogen bond. Since the steric conditions probably do not vary significantly from one type of polymer to the other, the reason may lie in a co-operative effect, in which the acetamido hydrogen bonds join with the glucosamine $\mathrm{C}_{(4)} \mathrm{OH} \rightarrow$ uronate ring oxygen hydrogen bond (Atkins et al., 1980; Fig. 1) to reinforce the prerferred structure. This third, extra, hydrogen bond is not possible in the chondroitin sulphate series, because $\mathrm{C}_{(4)} \mathrm{OH}$ of galactosamine is axial, and hence the chain may be more flexible, resulting in less-stable acetamido hydrogen-bonding. N.m.r. evidence from dimethyl sulphoxide solutions of the synthetic disaccharides given in Table 1 (Heatley et al., 1981) indicates that the third hydrogen bond is indeed present in hyalobiuronate but not in $\mathrm{N}$-acetylchondrosinate.

Thus the downfield shift of Resonance II with respect to Resonance I in spectra obtained from dimethyl sulphoxide solutions of glycosaminogly- curonans may show how stable the relevant acetamido hydrogen bonds are, and hence how potentially important the hydrogen-bonded structures are in vivo. Although dimethyl sulphoxide is not a physiological milieu, the findings are entirely compatible with those obtained by less direct methods in aqueous solutions. The parallels between our results and the c.d. studies on hyaluronate oligomers and chondroitin, conducted in water, by Cowman et al. (1981) are very close. Both c.d. and n.m.r. spectra demonstrate the appearance of typical polymer features at the tetrasaccharide level in the hyaluronate oligomer series, and both sets of results agree in implying that acetamido interaction with $-\mathrm{CO}_{2}{ }^{-}$is not as pronounced in chondroitin as in hyaluronate, as discussed above. The n.m.r. of the acetamido methyl group in ${ }^{2} \mathrm{H}_{2} \mathrm{O}$ solutions (Scott \& Heatley, 1979), as well as periodate oxidation kinetics in $\mathrm{H}_{2} \mathrm{O}$, point to the validity of structures similar to those shown in Fig. 1 (Scott \& Tigwell, 1975,1978 ), implying that the hydrogen bonds seen in dimethyl sulphoxide solutions are present in $\mathrm{H}_{2} \mathrm{O}$, and hence must be of physiological significance (Scott et al., 1981).

\section{References}

Atkins, E. D. T., Meader, D. \& Scott, J. E. (1980) Int. J. Biol. Macromol. 2, 318-319

Bitter, T. \& Muir, H. M. (1962) Anal. Biochem. 4, 330-334

Cowman, M. K., Balazs, E. A., Bergmann, C. W. \& Meyer, K. (1981) Biochemistry 20, 1379-1385

Heatley, F., Scott, J. E. \& Casu, B. (1979) Carbohydr. Res. 72, 13-23

Heatley, F., Scott, J. E., Jeanloz, R. \& Walker-Nasir, E. (1981) Carbohydr. Res. in the press

Reissig, J. L., Strominger, J. L. \& Leloir, L. F. (1955) J. Biol. Chem. 217, 959-966

Schamper, T. J. (1974) Carbohydr. Res. 36, 233-237

Scott, J. E. (1960) Methods Biochem. Anal. 8, 145-197

Scott, J. E. (1980) in Biology of the Articular Cartilage in Health and Disease (Gastpar, H., ed.), pp. 49-53, F. K. Schattauer Verlag, Stuttgart and New York.

Scott, J. E. \& Heatley, F. (1979) Biochem. J. 181, 445-449

Scott, J. E. \& Tigwell, M. (1975) Biochem. Soc. Trans. 3, 662-664

Scott, J. E. \& Tigwell, M. (1978) Biochem. J. 173, 103-114

Scott, J. E., Heatley, F., Moorcroft, D., Olavesen, A. H. \& Casu, B. (1977) Upsala J. Med. Sci. 82, 152

Scott, J. E., Orford, C. R. \& Hughes, E. W. (1981) Biochem.J. 195, 573-581 